\begin{tabular}{|c|c|c|}
\hline $\mathrm{Z}$ e i $t$ & $\begin{array}{l}\text { Volumina } \mathrm{CO}_{2} \text { in } \\
10000 \text { Vol. Luft. }\end{array}$ & $0 \mathrm{rt}$ \\
\hline $\begin{array}{l}\text { 8. März 1890, } 121 / 2 \text { Uhr Mittags. } \\
\text { 9. März } 1890,121 / 2 \text { Uhr Mittags. } \\
\text { 18. April 1890, } 21 / 2 \text { Uhr Nachmittags. } \\
\text { 18.-19. April } 1890,12 \text { Uhr Nachts. } \\
\text { 19.-20. April 1890, } 12 \text { Uhr Nachts. } \\
\text { 20. April 1890, } 4 \text { Uhr Nachmittags. } \\
\text { 23. April 1890, 4 Uhr Nachmittags. }\end{array}$ & $\begin{array}{l}2,88 \\
3,01 \\
2,98 \\
3,34 \\
3,00 \\
3,15 \\
2,95\end{array}$ & $\begin{array}{l}\text { Stadtgarten } \\
\text { (in der Stadt). } \\
\left\{\begin{array}{l}\text { Maly-Fentan } \\
\text { (Umgebung am } \\
\text { Schwarzen } \\
\text { Meer.) }\end{array}\right.\end{array}$ \\
\hline
\end{tabular}

Als Mittel der Beobachtungen kann man 3,04 Volumina $\mathrm{CO}_{2}$ auf 10000 Vol. Luft annehmen. Das Minimum ist gleich 2,88, das Maximum 3,34. Nach der beschriebenen Methode beabsichtige ich im nächsten Sommer eine grössere Anzahl von Bestimmungen auszuführen.

Ich erlaube mir an dieser Stelle Herrn Professor Dr. A. Werigo für die Unterstützung, die er mir bei Ausführung dieser Arbeit hat zu Theil werden lassen, meinen aufrichtigsten Dank abzustatten.

O dessa, Chemisches Laboratorium der Universität, November 1890.

\title{
Kohlensänrebestimmung.
}

$$
\text { Von }
$$

\section{Dr. F. Tschaplowitz,}

Chem. Leiter der Versuchşstation des Königl. pomologischen Institutes zl: Proskau.

Bei physiologischen Untersuchungen - Aschenanalysen - steht oft nur eine geringe Menge Material zur Verfügung. Ich führte deswegen die Kohlensäurebestimmungen nach der Methode von Franz Schulze*) aus, welche darin besteht, dass die entwickelte Kohlensäure in titrirtes Barytwasser geleitet und alsdann nach einigem Stehen darin mittelst Oxalsäure unter Anwendung von Curcumatinctur und -Papier auf bekannte Weise gemessen wird. Es findet sich jedoch, dass dabei das verlangte langsame Tempo der Entwicklung schwer einzuhalten ist und oft, besonders wenn das Kochen etwas stossweise erfolgt, nicht alle Kohlensäure vollständig absorbirt wird.

*) Diese Zeitschrift $\mathbf{9}, 290$. 
Wendet man andere Methoden, namentlich andere Absorptionsmittel - Natronkalk, Kalihydrat, Kalilauge - an, um durch deren Gewichtszunahme die Quantität der Kohlensäure zu ermitteln, so wird der durch das Erhitzen gleichzeitig mit entwickelte Wasserdampf von den zwischengeschalteten Substanzen - Schwefelsäure, Chlorcalcium, getrocknetes Kupfersulfat - nicht immer vollständig aufgenommen und dann theilweise als Kohlensäure gewogen. Entwickelt man dabei die Kohlensäure mittelst Salzsäure, so entweicht auch diese zum Theil, das dann einzuschaltende Kupfersulfat absorbirt aber dieselbe auch nicht immer vollständig.

Fig. 15.

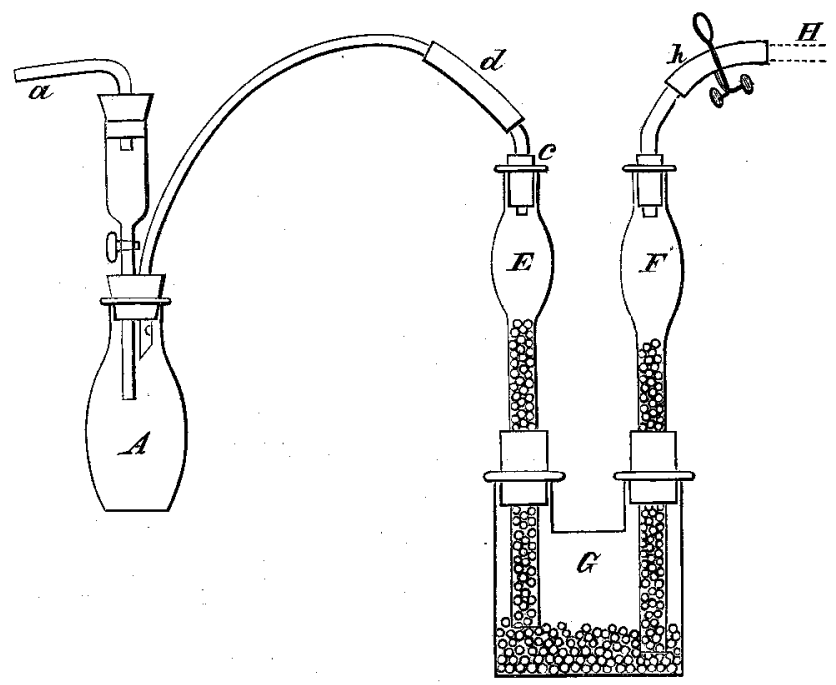

Bei den eben genannten Methoden, noch mehr aber bei denjenigen, bei welchen ein compendiöser Apparat gewogen werden muss, um durch die Gewichtsdifferenz die ausgetretene Kohlensäure za bestimmen, tritt noch der Fehler der Oberflächenverdichtung hinzu, insofern als diese Apparate wegen ihrer vielfach gekrümmten Oberflächen sich nicht leicht vollkommen rein oder auch nur in gleichem Oberflächenzustand erhalten lassen.

Zur Umgehung dieser Schwierigkeiten wende ich mit bestem Erfolg nachbeschriebenes Verfahren und den in Fig. 15 skizzirten Apparat an:

Die Kohlensäure wird mittelst Schwefelsäure ausgetrieben. Die dabei anzuwendende Koch- und Entwicklungsflasche muss genügend Raum bieten, um noch reichlich so viel (wohl ausgekochtes) Wasser 
aufnehmen zu können, dass, wenn die Substanz Kalk enthält, der sich bildende Gyps gelöst bleibt. Ich ziehe die bouteillenförmigen Entwicklungsflaschen, wie sie die Firma Chr. Kob\& Co., Stützerbach in Thüringen, liefert, den Rundkölbchen vor. Die Menge der Substanz wird so gewählt, dass sie $40-80 \mathrm{mg}$ Kohlensäure enthält. Nicht in Wasser lösliche Carbonate werden fein zerrieben in die Flasche gespült. Nach dem Einbringen des zu analysirenden Körpers führt man in den reinen, vorher mit ausgekochtem heissem Wasser ausgespülten Apparat durch Saugen bei a kohlensäurefreie Luft ein, öffnet bei c, lässt $25 c c$ Kalilauge von etwa 1,5\% Concentration, deren Titrirung auf die unten angegebene Weise ausgeführt ist, und etwa eben so viel Wasser ein, (so dass die auf dem Boden liegenden Glaskugeln nahezu bedeckt sind) und schliesst alsbald wieder.

Die Absorptionsflasche trägt zwei oben eiförmig erweiterte Röhren, welche bis zu dieser Erweiterung mit kleinen, etwa erbsengrossen Glaskugeln angefüllt sind; mit gleichen Kugeln ist auch der Boden der Flasche $4 \mathrm{~cm}$ hoch bedeckt. Das Rohr $\mathbf{E}$ steht etwas höher als $\mathrm{F}$, welches bei $\mathrm{H}$ mit einer Kalilauge enthaltenden, in der Fig. 15 nicht gezeichneten Schutzflasche verbunden ist. Man schliesst nun den Quetschhahn bei H, saugt ziemlich stark bei a, schliesst den Hahn der Trichterröhre schnell, um alsdann ein genügendes Quantum Schwefelsäure vorsichtig so, dass keine Luft mit eindringt, zu der in ausgekochtem Wasser gelösten oder vertheilten Substanz einfliessen lassen zu können. Hierauf erwärmt man langsam bis zum vollen Kochen. Die entwickelte Kohlensäure wird in G, selbst bei etwas stürmischer Entwicklung vollkommen absorbirt. Während der Operation kann die Absorptionsflasche etwas schief gestellt werden, im Anfang wird die in der Fig. 15 links befindliche, gegen Ende die rechts befindliche Seite etwas höher gestellt, aus leicht ersichtlichem Grunde, auch wird die Flamme unter A einigemal entfernt, um etwa nach $\mathbf{F}$ getretene Kohlensäure zurückzubringen, endlich auch G öfter etwas schüttelnd bewegt, soweit die Gummischlauchverbindungen bei $d$ und $h$ erlauben.

Die Form der Absorptionsflasche kann vielfach variirt werden. Mit ganz gleichem Erfolge habe ich Röhren angewendet, in welche mehrere andere engere Röhren concentrisch eingeschoben waren; indessen ist das Auswaschen derselben nicht bequem.

Nach Beendigung der Destillation werden die Kugeln aus $\mathrm{E}$ und $\mathbf{F}$ mittelst ausgekochten, noch heissen Wassers nach G gespült, überschüssige 
Chlorbaryumlösung (5 cc mit $0,8-1,0 \mathrm{~g} \mathrm{BaCl}$ ) zugesetzt, umgeschüttelt, (nach dem Verschliessen der beiden Oeffnungen) zwei Stunden bei Seite gestellt und endlich in derselben Flasche $(G)$ auf die bekannte Weise mittelst Oxalsäure $\left(1 /{ }_{20}\right.$ normal) unter Anwendung von Curcumatinctur und gutem Curcumapapier genau titrirt. Auf gleiche Weise ist der Titer der Kalilauge nach Zusatz einer gleichen Menge von Chlorbaryum and etwa $100 c c$ reinen, heissen Wassers nach zweistündiger Einwirkung vorher festzustellen.

Dass der Absorptionsapparat sammt den Glaskugeln vor dem Gebrauch mit Säure behandelt werden muss, ist eine wohl nicht zu erlassende Sicherheitsmaassregel.

Den Apparat liefert in vortrefflichster Ausführung die Firma $\mathrm{Chr}$. Kob \& Co. in Stützerbach in Thüringen.

Belege :

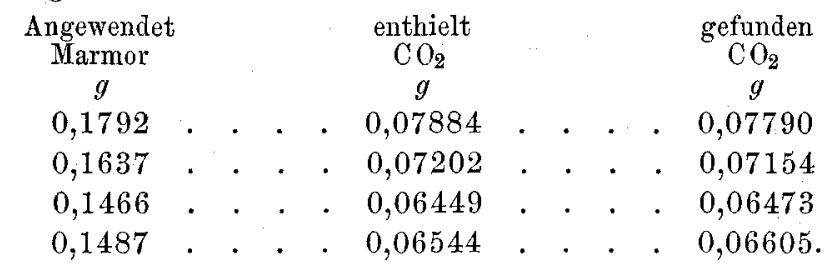

Natriumcarbonat $(2,1291 \mathrm{~g}$ ergaben nach dem Erhitzen $1,8141 \mathrm{~g})$ $10,0 \mathrm{~g}$ gelöst in $500 c c$, davon zur Bestimmung $10 \mathrm{cc}$, erforderten Oxalsäure:

$c c$

$\left.\begin{array}{ll}\text { 1) } & 32,0 \\ \text { 2) } & 32,5 \\ \text { 3) } & 32,0 \\ \text { 4) } & 31,75 \\ \text { 5) } & 32,2\end{array}\right\}$ anstatt $32,201 c c$.

Ueber Manganammoniumferrocyanür. Von

L. Blum.

Versetzt man die saure Lösung eines Mangansalzes mit Kaliumferrocyanür, so wird Manganferrocyanür abgeschieden nach der Gleichung:

$\mathrm{K}_{4} \mathrm{Fe}(\mathrm{CN})_{6} .3 \mathrm{H}_{2} \mathrm{O}+2 \mathrm{Mn} \mathrm{Cl}_{2}=\mathrm{Mn}_{2} \mathrm{Fe}(\mathrm{CN})_{6}+4 \mathrm{~K} \mathrm{Cl}+3 \mathrm{H}_{2} \mathrm{O}$.

Unter Umständen kann auch ein saures Mangansalz ausgefällt werden, das heisst eine Verbindung, in welcher nur ein zweiwerthiges Mangan- 